ज्) FRANÇAISE

$>\mathrm{DE}$

띨 PÉDAGOGIE

\section{Revue française de pédagogie}

Recherches en éducation

178 | janvier-mars 2012

Les politiques de lutte contre les inégalités scolaires d'un pays à l'autre

\title{
Un réseau d'enseignement prioritaire dans le canton de Genève : quels effets sur les élèves?
}

What are the effects of a priority education network in Geneva on student achievement?

Una red de enseñanza prioritaria en el Cantón de Ginebra: ¿cuáles fueron los efectos en los alumnos?

Ein schulpolitisches Fördernetz im Genfer Kanton: welche Auswirkungen auf die Schüler?

Anne Soussi, Christian Nidegger, Marion Dutrévis et Marcel Crahay

\section{OpenEdition}

\section{Journals}

Édition électronique

URL : http://journals.openedition.org/rfp/3548

DOI : $10.4000 /$ rfp.3548

ISSN : 2105-2913

Éditeur

ENS Éditions

Édition imprimée

Date de publication : 15 mars 2012

Pagination : 53-66

ISBN : 978-2-84788-372-5

ISSN : 0556-7807

Distribution électronique Cairn

CAIRN

CHERCHER, REPÉRER, AVANCER.

Référence électronique

Anne Soussi, Christian Nidegger, Marion Dutrévis et Marcel Crahay, « Un réseau d'enseignement prioritaire dans le canton de Genève : quels effets sur les élèves ? », Revue française de pédagogie [En ligne], 178 | janvier-mars 2012, mis en ligne le 15 mars 2015, consulté le 06 mai 2019. URL : http:// journals.openedition.org/rfp/3548; DOI : 10.4000/rfp.3548 


\section{Un réseau d'enseignement prioritaire dans le canton de Genève : quels effets sur les élèves?}

\section{Anne Soussi, Christian Nidegger, Marion Dutrévis et Marcel Crahay}

La lutte contre l'échec scolaire est en débat permanent à l'école, et ce quel que soit le pays. Depuis de nombreuses années, certains pays européens ont mis en place des politiques d'éducation compensatoire inspirées de celles introduites aux États-Unis dès les années soixante. En 2006, le canton de Genève a mis en place une politique de ce type. Après avoir donné un aperçu de la situation des politiques d'éducation prioritaire en Europe et de leurs effets, nous décrirons le réseau d'enseignement prioritaire genevois (REP par la suite) et ses spécificités. Dans un deuxième temps, nous tenterons de mesurer ses effets sur les acquis des élèves en comparant les élèves du REP à d'autres provenant d'établissements comparables non REP. Un groupe témoin d'établissements présentant les mêmes caractéristiques que les écoles REP a été constitué, ceci afin de se rapprocher du schéma des études quasi expérimentales. Des analyses multiniveaux ont été réalisées sur différentes mesures de performances scolaires. Conformément à ce que montre la littérature existante, les analyses ne permettent pas de mettre en évidence des effets de cette politique sur les acquis des élèves mais plutôt sur le climat scolaire.

Mots-clés (TESE) : zone d'éducation prioritaire, discrimination positive, échec scolaire, inégalité sociale, enseignement primaire.

La plupart des pays cherchent à lutter contre l'échec scolaire. Cette lutte est en débat permanent à l'école et s'inscrit dans la recherche d'une certaine justice. À ce sujet, trois principes peuvent être pris en compte (Crahay, 2000) : le principe d'égalité des chances qui repose sur la revendication méritocratique permettant de donner les mêmes chances de formation à des élèves d'égale aptitude, un principe qui s'accommode bien de l'existence de filières de valeur inégale ; celui d'égalité de traitement qui a pour principe d'offrir la même qualité d'enseignement à tous les élèves, et enfin celui d'égalité des acquis qui vise cet objectif par la mise en place de procédures de discrimination positive. Ce dernier principe (qui accepte des inégalités de résultats par delà les compétences jugées essentielles) peut passer par des inégalités de traitement à condition qu'elles soient motivées par une volonté de justice correctrice. C'est ce dernier principe qui anime les politiques d'éducation prioritaire (PEP). Après une brève description des PEP en Europe, le réseau d'enseignement prioritaire genevois (REP) et ses spécificités feront l'objet d'une présentation avant de mesurer les effets de cette politique sur les acquis des élèves. 


\section{LA SITUATION DES PEP EN EUROPE}

Depuis de nombreuses années, certains pays européens ont mis en place des politiques inspirées des politiques d'éducation compensatoire introduites aux États-Unis ${ }^{1}$ dès les années soixante. Demeuse, Frandji, Greger et alii ${ }^{2}$ (2008) les définissent comme des " politiques visant à agir sur un désavantage scolaire à travers des dispositifs ou des programmes d'action ciblés (que ce ciblage soit opéré selon des critères ou des découpages socio-économiques, ethniques, linguistiques ou religieux, territoriaux ou scolaires) en proposant de donner aux populations ainsi déterminées quelque chose de plus (ou de "mieux" ou de "différent") " (Demeuse, Frandji, Greger et al., 2008, p. 12). Ces politiques peuvent suivre une logique territoriale ou se focaliser sur des populations ciblées (groupes à risque, enfants à besoins spécifiques ${ }^{3}$, etc., voir Dutrévis \& Crahay, 2009). Rochex (2010) distingue trois « âges » des PEP : le premier répond à une logique purement compensatoire, le second se caractérise par l'autonomie des établissements et la diversification de l'offre scolaire qui pourrait être assimilée à un modèle de quasi-marché (compétition et évaluation des établissements) et le troisième met l'accent sur l'individualisation des parcours ou des apprentissages afin de rendre maximales les chances de réussite pour tous. Toutefois, dans la plupart des pays qui ont introduit ce type de politique, les effets se sont avérés décevants (Dutrévis \& Crahay, 2009 ; Rochex, 2008). Ainsi, dans une étude européenne sur les PEP, Rochex (2008) parle dans sa conclusion de «no evidence-based policies " pour déplorer le manque de données empiriques, ce qui devrait conduire les décideurs à interroger la perpétuation ou les différents modes d'action de ces politiques. Cette absence de conclusions claires sur les effets des PEP concernant la lutte contre l'échec scolaire et les inégalités tient, selon lui, à des réticences de la part des différents acteurs et, sur le plan méthodologique, à des données très variables selon les administrations, mais aussi et surtout à la difficulté à mettre en évidence ce qui est dû à ces PEP. II souligne en outre la fragilité de certains résultats, comme par exemple les effets bénéfiques constatés pour les jeunes enfants anglais de milieux défavorisés ayant bénéficié d'interventions précoces, qui s'estompent au fil des années. II n'exclut pas que des progrès aient été accomplis au niveau des établissements concernant la démocratisation de leur fonctionnement et l'accueil de la diversité des pratiques et des cultures, mais ces aspects ont - semble-t-il - peu fait l'objet d'évaluations externes.
En 1994, Meuret évoquait déjà, pour la France, des effets décevants des ZEP. II expliquait alors que les effets positifs de discrimination positive se situaient davantage au niveau du climat des établissements et des classes, ou encore au niveau de la socialisation des élèves, qu'au niveau des acquis scolaires (cf. Meuret, 1994, 2000). Toujours en ce qui concerne l'hexagone, le rapport de Moisan et Simon (1997) a mis en évidence la grande disparité des ZEP et montré des effets positifs observables de certaines d'entre elles. Les auteurs ont pu ainsi dégager quelques conditions favorables à l'efficacité des dispositifs d'éducation prioritaire, notamment l'engagement des équipes, les projets pédagogiques basés sur les savoirs des élèves et la fréquentation de l'école maternelle.

\section{LA SITUATION GENEVOISE : POURQUOI INTRODUIRE UN RÉSEAU D'ENSEIGNEMENT PRIORITAIRE ?}

À Genève, la problématique de l'échec scolaire et la lutte contre les inégalités sont une préoccupation depuis de nombreuses années ${ }^{4}$ (cf. notamment Hutmacher, 1993 ; Perrenoud, 1984). Toutefois ce n'est que récemment, avec l'augmentation de populations défavorisées dans certains quartiers et les difficultés croissantes ressenties par les enseignants, que s'est imposée la nécessité d'introduire une politique de discrimination positive ${ }^{5}$. II existait déjà un système d'allocation de ressources différenciées au secondaire inférieur (collège) selon la composition socio-économique des établissements, mais le pas a été franchi d'introduire progressivement, à l'école primaire dans un premier temps, une véritable politique d'éducation prioritaire sous forme d'un réseau d'écoles fréquentées par un taux particulièrement important d'élèves de milieu modeste. Forts des expériences nord-américaines, européennes et françaises en particulier, les responsables politiques genevois ont choisi un certain nombre d'options parmi celles considérées comme favorables : l'engagement des équipes enseignantes sur trois ans, l'élaboration de projets d'établissement, l'introduction de directions et de conseils d'établissements, l'accent mis sur les relations avec les familles, etc. Toutefois, un certain nombre des mesures mises en place dans le REP ont été par la suite généralisées à l'ensemble des établissements primaires du canton, comme c'est le cas des PEP, bien souvent terrains d'expérimentation des politiques éducatives et sociales. Le REP s'inscrit bien dans ce courant des PEP avec des établissements 
sélectionnés au niveau cantonal (par la direction de l'enseignement primaire) sur la base d'un critère principal (en l'occurrence, la proportion d'élèves provenant de milieux défavorisés) et d'un critère secondaire (la proportion d'élèves allophones).

Comme dans les autres pays européens ou nordaméricains, la politique d'éducation prioritaire mise en place à l'école primaire ${ }^{6}$ dès 2006 dans le canton de Genève a pour objectif de lutter contre l'échec scolaire et de tendre vers l'égalité des acquis pour tous les élèves, quel que soit leur milieu socio-économique. Ce type de mesure se justifie par un lien incontestable entre réussite scolaire et origine sociale, montré dans la plupart des études mesurant ces variables (constat classique dont l'universalité a été confirmée par les études internationales PISA). Cette mesure s'inscrit dans un projet plus vaste de réorganisation de l'école primaire (autonomie partielle des établissements, réorganisation des structures hiérarchiques avec notamment l'introduction de directions d'école, de conseils d'établissements, etc.). C'est pourquoi il est parfois difficile de distinguer ce qui est spécifique au REP des réformes plus générales du système scolaire. Le REP genevois s'est focalisé pour l'instant sur l'enseignement primaire. C'est à ce niveau d'enseignement qu'il a été jugé le plus urgent d'introduire de telles mesures. La littérature scientifique conforte largement cette position : c'est au début de la scolarité que se joue en partie la carrière scolaire d'un élève, que l'origine sociale est déterminante et que des interventions précoces sont bénéfiques pour donner à tous la même égalité des chances et prévenir l'échec scolaire (Crahay, 2000 ; Crahay \& Dutrévis, 2012). Les caractéristiques liées à la taille des écoles primaires, plus petites que celle des établissements du secondaire inférieur, d'une part, et, d'autre part, la présence d'enseignants engagés et volontaires, dont certains s'étaient investis précédemment dans d'autres projets de transformation de l'école (comme la rénovation de l'enseignement primaire), ont été également déterminantes. Le REP genevois, qui cible des établissements dans des quartiers défavorisés, relève surtout à notre avis d'une logique territoriale, c'est-à-dire plutôt du premier âge des PEP (modèle compensatoire où l'on donne plus à ceux qui ont moins) décrit par Rochex $(2008,2010)$. En effet il s'agit clairement d'une politique compensatoire s'adressant à des établissements comportant une forte proportion d'enfants de milieux défavorisés scolarisés dans certains établissements (cela relève également d'une logique territoriale).

Ce réseau d'enseignement prioritaire s'est constitué petit à petit depuis 2006 avec d'abord un seul établis- sement pilote particulièrement défavorisé du point de vue de la composition socio-économique de son public d'élèves, puis 6 autres l'année d'après, puis encore 7 autres en 2008. Lors de la dernière phase de prise d'informations, le REP en comptait donc 14 et s'est encore développé depuis puisqu'à la rentrée 2010, 17 établissements sur les 90 établissements primaires du canton de Genève (c'est-à-dire près de $20 \%$ d'entre eux) faisaient partie du réseau. Au vu des critères retenus, le REP ne devrait pas s'étendre à l'avenir, du moins dans l'enseignement primaire ${ }^{7}$. Pour faire partie du REP, les établissements doivent remplir principalement deux conditions : une proportion d'au moins $55 \%$ de parents d'élèves issus de catégories socioéconomiques défavorisées (la moyenne pour l'ensemble du canton se situant autour de $38 \%$ ) et l'engagement de l'ensemble de l'équipe enseignante pour une durée de 3 ans $^{8}$. À ces deux conditions s'ajoute une troisième, au second plan dans les textes, mais cruciale étant donné le rôle prépondérant et attesté que joue la langue dans l'acquisition des savoirs : il s'agit de la proportion d'élèves allophones supérieure à $60 \%$ (la moyenne cantonale se situant à $40 \%$ ). Alors que ce critère n'est pas considéré comme prioritaire pour décider l'entrée d'une école dans le réseau, la maîtrise de la langue et de l'écrit constitue une priorité dans la plupart des projets d'école : il faut relever à cet égard une sorte de contradiction.

Les établissements faisant partie du REP bénéficient d'un certain nombre de mesures particulières : un peu plus de ressources allouées, une plus grande autonomie grâce à la présence de directions de proximité et de projets d'établissement, des actions coordonnées entre établissements et professionnels de l'Office de la jeunesse, des effectifs de classe allégés, la présence d'un éducateur jouant le rôle de coordinateur entre écoles et familles, l'implication des communes, les liens renforcés avec les parents... Ces différents éléments avaient pour but de créer les meilleures conditions possible au niveau du taux d'encadrement et du climat scolaire pour favoriser les conditions d'apprentissage de tous les élèves.

Les premiers résultats observés à Genève mettaient en évidence des effets encourageants de la mise en place du REP au niveau du fonctionnement et de l'organisation. Ainsi Jaeggi et Osiek $(2007,2008)$ ont pu montrer des effets positifs de l'introduction de directions et surtout d'un éducateur sur le climat de l'école ${ }^{9}$. En particulier, les éducateurs ont été estimés par l'ensemble des acteurs de l'école comme ayant un rôle particulièrement important dans l'aide aux élèves en difficulté. Ces résultats vont dans le même 
sens que ceux des études réalisées en France sur les ZEP (Meuret, 1994 ; Moisan \& Simon, 1997, notamment) ou en Europe en général (Demeuse, Frandji, Greger et al., 2008). Par ailleurs, l'observation des connaissances des élèves de l'établissement pilote (établissement se caractérisant par un nombre particulièrement important d'élèves de milieux défavorisés et d'allophones) au moment de leur entrée à l'école (4 ans) a montré que leurs connaissances initiales dans le domaine du langage et des mathématiques ${ }^{10}$ étaient plus faibles que celles d'élèves scolarisés hors REP, surtout quand elles faisaient appel à des compétences langagières, mesurées aussi dans le domaine mathématique. Toutefois elles n'étaient pas fondamentalement différentes d'un point de vue qualitatif de celles d'une population de référence (Saada, 2006 ; Soussi, Guignard, Hayoz et al., 2008). Autrement dit, les différences entre élèves du REP et élèves non REP varient largement en fonction des items examinés, et donc des compétences spécifiques évaluées. Ainsi, les élèves sont parvenus sans difficulté à réaliser certaines tâches (compréhension de consignes orales, connaissance et écriture de certaines lettres et de leur prénom) comme leurs homologues des écoles non REP. D'autres tâches leur ont posé davantage problème comme la connaissance du vocabulaire ; à cet égard, ils se situent en dessous de la moyenne d'une population du même âge. Ces écarts sont sans doute explicables par la présence importante d'élèves allophones. On a pu constater également la présence d'un certain nombre de connaissances en mathématiques tout à fait comparables à celles d'élèves du même âge scolarisés dans d'autres établissements du canton (comptage et dénombrement de 10 jetons, lecture et écriture de chiffres). En ce qui concerne les compétences de raisonnement non verbal (sous-test WPPSI-III), nous n'avons pas non plus constaté de différences par rapport à une population tout-venant du même âge. Ces résultats vont dans le même sens que ceux d'une étude comparative réalisée en France auprès d'élèves de grande section de maternelle (Bara, Gentaz \& Colé, 2008), montrant des résultats moins bons pour les élèves scolarisés dans des classes REP comparativement à leurs camarades scolarisés dans des classes régulières dans des tâches de vocabulaire, de conscience rimique ou de connaissance de lettres, mais pas dans un test de QI non verbal.

Dans l'étude genevoise, l'observation de l'entrée dans le métier d'élève au moyen d'une grille remplie par les enseignants a montré que les élèves de REP possédaient des acquis proches de ceux d'une popu- lation de référence, mais qu'il y avait également des différences au niveau des règles de vie de la classe, des relations avec les enseignants ou avec les pairs ou encore des attitudes face aux activités et au travail scolaire. Ces différences peuvent notamment s'expliquer par la fréquentation ou non d'une institution de la petite enfance (crèche ou jardin d'enfants). À la fin de la première année d'école (école maternelle), la majorité des élèves a atteint les objectifs attendus au niveau de l'apprentissage des règles de vie de la classe, des relations avec les enseignants ou encore des attitudes par rapport au travail scolaire ${ }^{11}$, ce qui est encourageant étant donné la grande proportion d'élèves qui possèdent une culture familiale relativement éloignée de celle de l'école, non seulement au niveau de la langue parlée mais également des pratiques de l'écrit. On connaît effectivement la difficulté des enfants à entrer dans le métier d'élève, qui suppose l'intégration d'un certain nombre de règles propres à la vie scolaire, qui font ainsi passer du statut d'enfant à celui d'élève (notamment Sirota, 1993 ; Chauveau, 2000), une difficulté qui est accrue lorsque la culture familiale diffère de celle de l'école.

La lecture constitue également un domaine crucial de par son rôle majeur dans l'acquisition des connaissances scolaires. C'est pourquoi elle a aussi fait l'objet d'études au moyen d'un test au début et à la fin de la première année primaire (l'équivalent du CP français, c'est-à-dire pour des élèves de 6-7 ans). L'objectif était de comparer, au début de l'apprentissage de l'écrit, les compétences des élèves du REP et celles d'élèves d'établissements témoins se situant dans la moyenne cantonale du point de vue de deux caractéristiques sociodémographiques (proportions d'enfants de milieux défavorisés et d'élèves allophones). En définitive, les chercheurs ont pu observer des différences entre les élèves des deux types d'établissement testés en début mais pas en fin d'année, en faveur des élèves scolarisés dans des établissements hors REP (Soussi \& Nidegger, 2010). Par ailleurs, la fréquentation d'une institution de la petite enfance (crèche, jardin d'enfants, etc.) est, comme dans le cas de la socialisation, un facteur déterminant des compétences en lecture au début de la $1^{\text {re }}$ primaire ; ceci corrobore l'une des constatations du rapport de Moisan et Simon (1997) concernant les « bonnes » ZEP. En fin d'année, le score initial détermine davantage les compétences en lecture que la fréquentation d'une institution de la petite enfance ${ }^{12}$. Ce résultat va dans le même sens que celui d'autres études (voir par exemple Duru-Bellat, Le Bastard-Landrier, Piquée et al., 2004 ; Piquée, 2010). 


\section{LE REP GENEVOIS : UN RÉSEAU HÉTÉROGÈNE}

De manière générale, on observe une certaine diversité parmi les 14 établissements du REP genevois, et ceci du point de vue des quatre critères pris en compte pour décrire les établissements (taille de l'établissement, pourcentage d'enfants de milieux défavorisés, pourcentage d'élèves allophones, pourcentage d'élèves en retard scolaire $\left.{ }^{13}\right)$. Pour la plupart de ces critères, on observe une importante variation entre établissements : la proportion d'enfants de milieux défavorisés (parents entrant dans la catégorie " ouvriers et divers/sans indication ") varie entre $49 \%$ et $69 \%$ pour l'année considérée (2008-2009) alors qu'il s'agit de la condition principale d'entrée dans le REP. Bien plus, la règle précise que la proportion d'enfants de milieux défavorisés doit être d'au moins $55 \%$. Or cinq établissements n'atteignent pas ce seuil. La proportion d'élèves allophones varie quant à elle entre $37 \%$ et $73 \%$. Plusieurs établissements ont une proportion élevée pour ces deux critères (proportion d'enfants de milieux défavorisés supérieure à $55 \%$ et d'élèves allophones supérieur à $60 \%$ ), alors que d'autres se situent dans la moyenne cantonale. Par ailleurs, quand on considère ce second paramètre dans l'ensemble des établissements genevois, il ressort que certains établissements hors REP présentent des taux d'élèves allophones équivalents à ceux du REP. Toutefois, si l'on compare les établissements REP à la moyenne des établissements genevois, ils comportent tous une plus grande proportion d'élèves de milieux défavorisés et d'élèves allophones, la proportion cantonale moyenne étant de $37 \%$ pour le premier critère et de $42 \%$ pour le second, alors qu'elle est respectivement de $57 \%$ et de $56 \%$ dans l'ensemble du REP.

Il est par ailleurs intéressant de noter que la proportion d'élèves en retard scolaire au sein des établissements REP est également variable. Si l'on regarde plus spécifiquement ce qu'il en est pour l'établissement pilote qui présente la situation extrême, on constate que la proportion d'élèves en retard est à peine plus élevée (9\%) que celle de l'ensemble des 90 établissements du canton (8\%). Dans trois établissements REP, cette proportion est même inférieure à celle de l'ensemble du canton. En revanche, dans sept autres, la proportion d'élèves en retard est supérieure à $10 \%$. En définitive, si l'on prend en compte à la fois la proportion d'élèves de milieux modestes ainsi que celle d'allophones pour l'ensemble du canton, on constate que,

Figure 1. Établissements primaires du canton de Genève en fonction de la proportion d'enfants de milieux défavorisés et d'élèves allophones (en 2009)

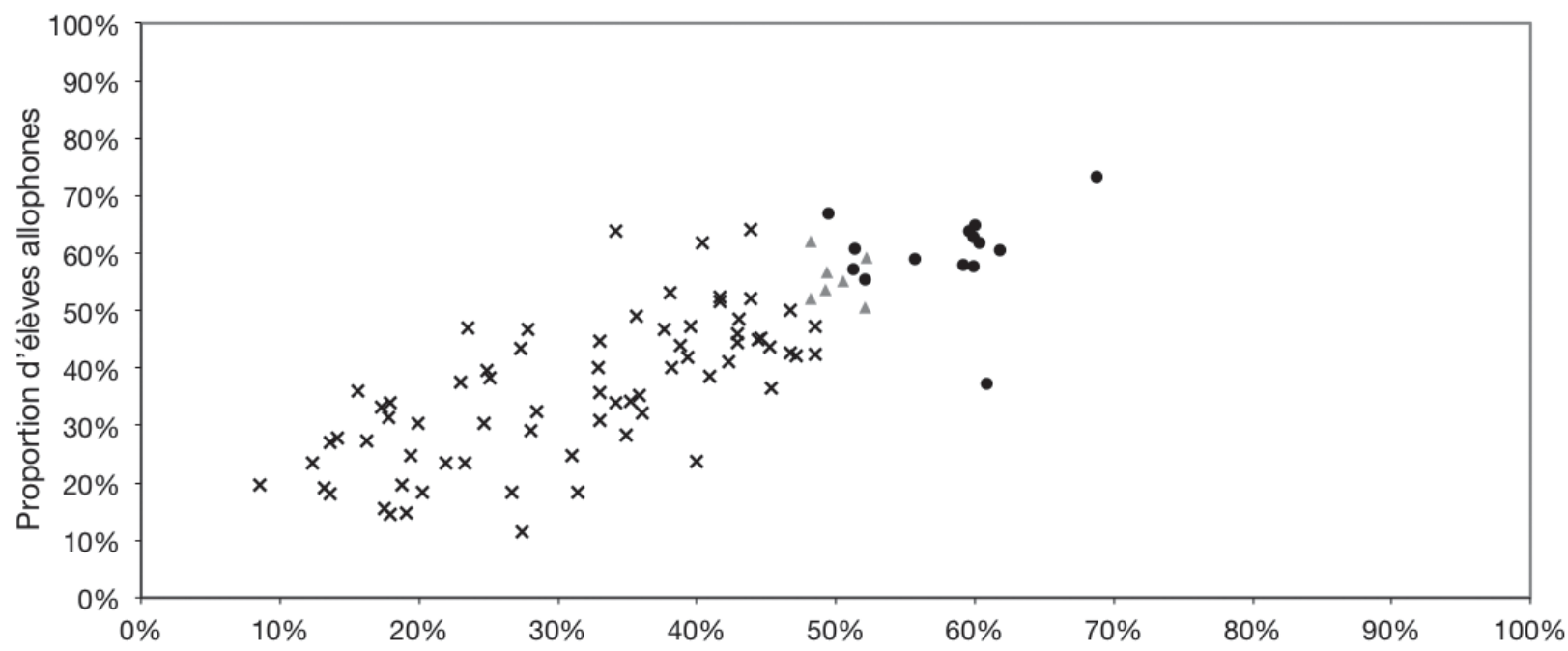

Proportion d'enfants d'“ ouvriers et divers/sans indication »

•Établissements REP × Établissements hors REP A Établissements témoins 
même si les établissements du REP sont relativement hétérogènes, ils se différencient malgré tout passablement des autres établissements concernant ces caractéristiques (cf. la figure 1). Par contre, quelques établissements qui ne se trouvaient pas dans le REP au moment de l'étude sont relativement proches du point de vue des deux critères considérés. Ce sont ces établissements que nous allons utiliser comme groupe témoin.

\section{QUESTIONS DE RECHERCHE}

L'étude présentée dans cet article fait partie d'une évaluation plus globale des effets de la mise en place du REP. Celle-ci est mandatée au service de la recherche en éducation du canton de Genève par le département de l'instruction publique et organisée autour de deux axes : l'un traitant du fonctionnement, l'autre traitant des apprentissages des élèves. Dans le présent article, nous nous centrerons essentiellement sur le second volet. Comme dans la majorité des pays qui ont mis en place une politique de ce type, la principale question que nous nous posons est de savoir si le REP a des effets positifs sur les acquis des élèves. En d'autres termes, l'hypothèse est la suivante : les établissements ayant bénéficié de mesures particulières liées au REP réussissent mieux que ceux ayant des caractéristiques comparables et qui n'en ont pas bénéficié. Dans la perspective qui vient d'être décrite, nous avons donc constitué un groupe témoin d'établissements présentant des caractéristiques quasi équivalentes à celles du REP, ceci du point de vue des deux critères principaux : la proportion d'élèves de milieux défavorisés et d'élèves allophones.

Comme dans la plupart des pays, les résultats des élèves sont souvent liés à un certain nombre de caractéristiques sociodémographiques (genre, âge, langue parlée à la maison, milieu socio-économique, etc.). De ce fait, on peut supposer que ces caractéristiques ont une influence certaine sur la réussite du programme scolaire déterminée par des objectifs. Les mesures entreprises dans le REP devraient permettre une discrimination positive en compensant les lacunes et les écarts des élèves concernés par rapport à d'autres élèves mieux dotés, grâce à un certain nombre d'éléments (moins d'élèves par classe, plus d'encadrement, aides, etc.) et ainsi diminuer les différences et faire en sorte qu'une plus grande proportion d'élèves réussissent les objectifs du programme. Au départ, les mesures introduites dans les établissements REP sont davantage centrées sur l'environnement scolaire. Elles ont pour but d'améliorer les conditions d'enseignement afin de donner à tous les élèves les meilleures chances et donc devraient également avoir des effets sur les résultats scolaires. Dans la méthodologie expérimentale proposée, on cherche à contrôler l'effet de l'origine sociale en considérant des écoles comparables à ce niveau, les unes recevant des mesures améliorant les conditions d'enseignement. Ainsi les effets observés ne pourront être imputés à la composition sociale des établissements, mais bien à l'inscription ou non des établissements dans le REP, et donc aux moyens supplémentaires alloués à ces établissements.

\section{MÉTHODES ET INSTRUMENTS UTILISÉS}

Dans une étude précédente, Soussi, Guignard, Hayoz et alii (2008) et Soussi et Nidegger (2010) ont comparé les "rendements » des établissements du REP à celui de l'ensemble des autres établissements du canton et observé des différences de performance défavorables aux établissements REP par rapport aux autres établissements du canton. Ce résultat était en quelque sorte prévisible puisqu'étaient comparés des établissements aux caractéristiques particulières avec d'autres du tout-venant. En nous centrant sur les établissements REP et des établissements comparables du point de vue des deux caractéristiques principales d'intégration dans le réseau, nous avons l'ambition d'opérer une comparaison de type quasi expérimentale entre les établissements REP et non REP, toutes choses égales par ailleurs. À composition d'élèves comparable (les uns scolarisés dans des établissements bénéficiant de mesures de discrimination positive, les autres non), quel est l'effet des mesures REP sur les résultats des élèves ? Bien sûr, nous sommes encore loin de cet idéal, car il est évident que la condition ceteris paribus est loin d'être réalisée dans la comparaison décrite ici. Néanmoins, on peut considérer que cette comparaison peut mieux rendre compte de l'effet de l'intégration d'un établissement dans le REP que la comparaison opérée dans l'étude précédente.

Pour constituer le groupe témoin, nous avons choisi parmi les 90 établissements genevois ceux qui se trouvent dans le tiers supérieur de la distribution pour les deux critères retenus (établissements avec des taux élevés d'élèves de milieux défavorisés et allophones). Au total, parallèlement aux 14 établissements du groupe expérimental, un groupe de 
Figure 2. Réussite dans les trois domaines étudiés en $2^{\mathrm{e}}, 4^{\mathrm{e}}$ et $6^{\mathrm{e}}$ années primaires selon le type d'établissement (canton de Genève)

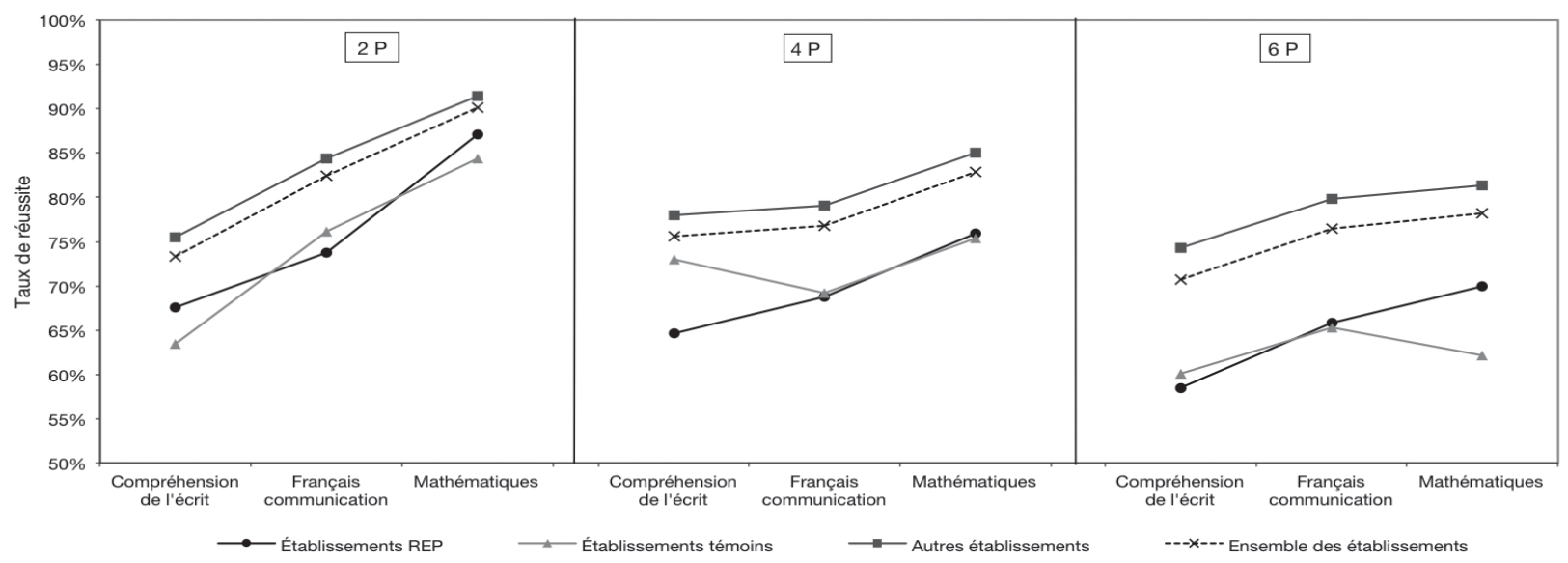

7 établissements témoins (comportant une proportion d'au moins $48 \%$ d'enfants dont les parents entrent dans la catégorie " ouvriers et divers/sans indication" ainsi que d'élèves allophones) a pu être constitué ${ }^{14}$. Notons qu'à titre indicatif, nous fournirons également les résultats des établissements hors REP. En ce qui concerne les variables dépendantes, il convient de signaler que notre évaluation des effets de l'intégration en REP sur les compétences des élèves se base principalement sur l'analyse des résultats aux épreuves externes cantonales. Ces évaluations sont soumises à tous les élèves d'une volée à trois moments du degré primaire : en $2^{\mathrm{e}}$ année primaire ${ }^{15}$ (7-8 ans), en $4^{\mathrm{e}}$ année primaire (9-10 ans) et 6e année primaire (11-12 ans) en français et mathématiques, ainsi qu'en allemand dès la $4^{\mathrm{e}}$ année primaire.

\section{RÉSULTATS DES DIFFÉRENTES COMPARAISONS}

Nous allons maintenant présenter les résultats les plus saillants des comparaisons opérées entre élèves scolarisés dans les établissements du REP, les établissements témoins ou les autres établissements.

\section{Résultats aux évaluations externes en français et mathématiques en $2^{\mathrm{e}}, 4^{\mathrm{e}}$ et $6^{\mathrm{e}}$ années primaires}

Comme annoncé auparavant, les évaluations externes constituent l'instrument principal utilisé pour observer les effets du REP. Nous allons maintenant présenter les principaux résultats des analyses réalisées sur les évaluations externes dans les deux groupes d'établissements, en nous centrant plus particulièrement sur trois domaines disciplinaires : la compréhension de l'écrit, la structuration (français structuration) et les mathématiques dans les trois degrés considérés pour l'année 2008-200916. Si les résultats des élèves des établissements du REP aux évaluations externes se différencient systématiquement de ceux d'élèves des établissements hors REP, qu'en est-il lorsque l'on compare les élèves des établissements REP et d'établissements présentant des caractéristiques comparables du point de vue de la composition sociodémographique ? Lorsque l'on examine la figure 2, on peut constater des écarts de réussite entre élèves scolarisés dans le REP et ceux scolarisés dans les établissements hors REP, quels que soient le degré ou la discipline. Par contre, le taux de réussite des élèves scolarisés dans les établissements témoins est proche de ceux provenant du REP.

Si l'on tient compte de certaines critiques faites à ce type de comparaison et que l'on prend en compte la proportion d'élèves de milieux défavorisés, on peut faire d'autres constats en termes de valeur ajoutée, comme l'illustrent les figures 3 et 4 pour la compréhension de l'écrit. Nous avons choisi de nous centrer ici sur les résultats en compréhension de l'écrit (plus particulièrement au début de l'apprentissage de la lecture et à la fin de l'école primaire) dont on connaît l'effet sur les apprentissages scolaires, mais le même type d'observation peut être effectué pour les mathématiques. Quand on calcule la valeur ajoutée ${ }^{17}$, un certain nombre d'établissements obtient un taux de réussite moyen en compréhension de l'écrit en $2^{\mathrm{e}}$ ou $6^{\mathrm{e}}$ année primaire supérieur à ce que l'on pouvait 
attendre, compte tenu de la proportion d'enfants dont les parents entrent dans la catégorie " ouvriers et divers/sans indication " dans ces établissements. Si l'on observe plus précisément à l'intérieur de chaque ensemble (établissements se situant dans le REP en 2008-2009, établissements témoins et établissements hors REP), la situation est un peu différente (cf. figures 3 et 4). En $2^{\mathrm{e}}$ année primaire, 5 établissements du REP sur 14 (c'est-à-dire moins de la moitié) ont une valeur ajoutée positive, c'est-à-dire qu'ils obtiennent un meilleur taux de réussite à l'épreuve qu'attendu.
La proportion est nettement plus faible encore dans les établissements témoins où un seul établissement sur 7 obtient un meilleur taux de réussite qu'attendu. Pour les établissements hors REP, la valeur ajoutée est positive pour 37 d'entre eux sur 59, c'est-à-dire deux tiers. En $6 \mathrm{e}$ année primaire, la situation est encore moins favorable pour les établissements du REP et les établissements témoins : 2 établissements du REP sur 14 ont une valeur ajoutée positive et un seul pour ce qui concerne les établissements témoins. Pour les établissements hors REP, le nombre d'établissements

Figure 3. Réussite observée et réussite attendue en compréhension de l'écrit en $2^{e}$ année primaire selon l'origine sociale des élèves (2008)

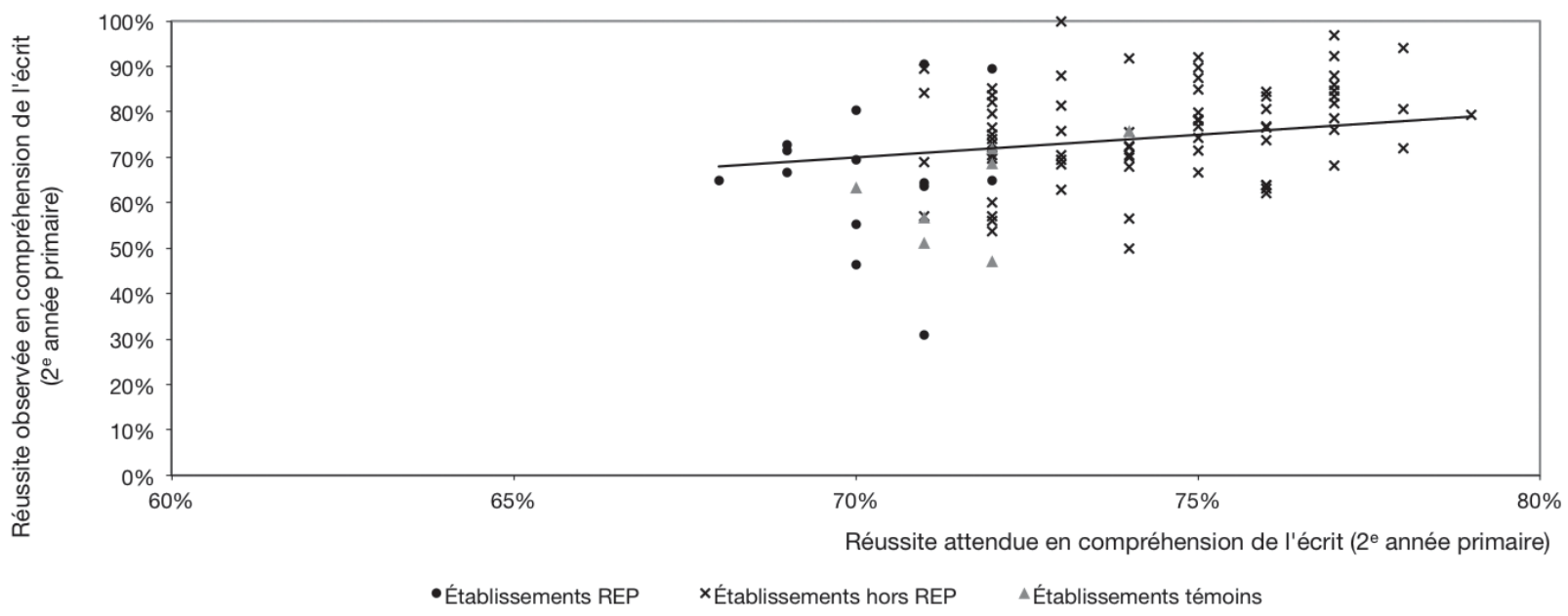

Figure 4. Réussite observée et réussite attendue en compréhension de l'écrit en 6 e année primaire selon l'origine sociale des élèves (2008)

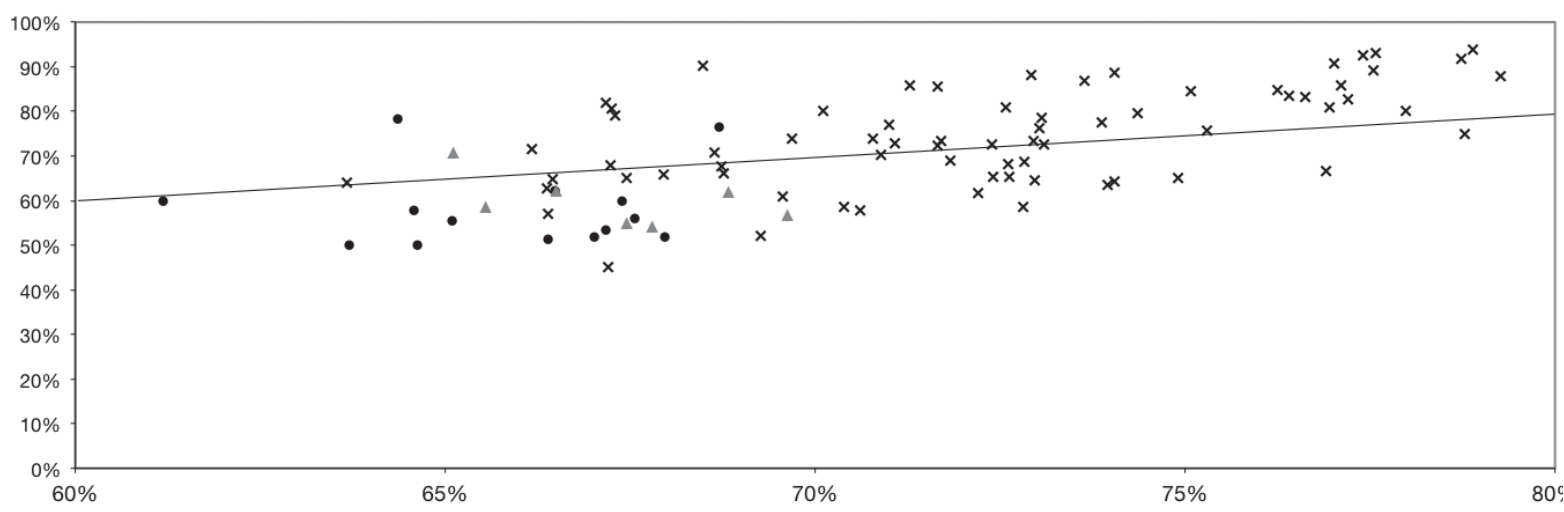

Réussite attendue en compréhension de l'écrit ( $6^{\mathrm{e}}$ année primaire)

•Établissements REP ×Établissements hors REP \Établissements témoins 
obtenant une valeur ajoutée positive est également inférieur à la situation en $2^{\mathrm{e}}$ année primaire : 37 sur 70 , c'est-à-dire à peine la moitié.

\section{Effet-classe ou effet-établissement?}

Afin de vérifier si le contexte, et plus particulièrement la scolarisation dans un établissement bénéficiant de mesures REP, avait un effet sur les performances des élèves, nous avons effectué tout d'abord une analyse de variance pour voir s'il existait des différences de performances entre établissements du REP, établissements du groupe témoin et autres établissements. II ressort de ces analyses que, dans la plupart des cas, il n'y a pas de différences significatives entre établissements REP et témoins. On observe une seule exception qui ne se révèle même pas au profit des élèves des établissements REP ; celle-ci concerne le français structuration en $4^{\mathrm{e}}$ année primaire. Quant aux élèves des autres établissements, ils ont systématiquement des résultats supérieurs et statistiquement différents de ceux des deux autres types d'établissement (REP et témoins), et ce quels que soient le domaine et le degré considérés. Pour aller plus loin dans l'observation des effets du contexte sur les performances des élèves, nous avons procédé à des analyses multiniveaux (élèves, classes, établissements) en nous limitant aux établissements REP et témoins. Voici les caractéristiques que nous avons pris en compte aux trois niveaux étudiés :

- au niveau " élèves » : le genre, la première langue parlée à la maison, l'origine socio-économique, l'âge ${ }^{18}$, le moment de l'arrivée dans la scolarité à Genève, le score initial, c'est-à-dire le résultat aux évaluations cantonales deux ans auparavant quand on en dispose ${ }^{19}$;

- au niveau " classes » : la taille de la classe, le type de classe, la proportion d'élèves allophones, la proportion de filles ou de garçons, celle d'enfants de milieux défavorisés, le niveau moyen initial de la classe dans la discipline considérée (quand il était disponible) ;

- au niveau " établissements " : la taille de l'établissement, le type d'établissement (REP ou témoin), le nombre d'années de fréquentation du REP, la proportion d'élèves allophones, la proportion d'enfants de milieux défavorisés, celle d'élèves en retard.

De manière générale, la question posée est la suivante : les différences de scores entre élèves sontelles attribuables à leurs caractéristiques individuelles, à celles des classes ou encore à celles des établisse- ments où ils sont scolarisés ? En d'autres termes, il s'agit de savoir si le poids de l'établissement fréquenté - REP ou non - est supérieur ou, au contraire, inférieur à celui des caractéristiques individuelles ou à celui de la classe fréquentée. Lorsqu'on décompose la variance en 3 niveaux, ce sont les caractéristiques individuelles (niveau "élèves") qui expliquent la plus grande part de variance (entre $78 \%$ et $98 \%$ ), les caractéristiques des classes qui expliquent entre 2 et $20 \%$ de variance et celle des établissements qui expliquent une faible part de variance, voire aucune (cf. le tableau 1). Les analyses multiniveaux mettent donc en évidence un rôle prépondérant joué par les caractéristiques individuelles des élèves, phénomène souvent montré dans les recherches de ce type (Bressoux, 1994, 2009 ; Dumay \& Dupriez, 2009).

Afin de vérifier la solidité de ce résultat, nous avons réalisé une analyse à deux niveaux (élèves et établissements) : nous conservons le niveau établissement afin d'évaluer au mieux les effets du REP sur les performances des élèves. De nouveau, dans la plupart des cas, la part de la variable expliquée par le niveau établissement est faible, ce qui confirme les résultats des analyses précédentes. On n'observe donc pas d'effet spécifique sur les performances du fait d'être scolarisé dans un établissement bénéficiant des mesures spéciales du REP, quels que soient le degré et la discipline considérés. Relevons toutefois que, lorsque l'on effectue des analyses à deux niveaux en considérant cette fois les élèves et les classes, on observe des différences entre classes plus ou moins marquées selon le degré ou la discipline considérés (en particulier en $2^{\mathrm{e}}$ année primaire). La composition de la classe, voire les pratiques pédagogiques, le style d'enseignement (frontal, interactif, etc., voir notamment Crahay, 2006) ou le nombre d'années d'expérience des enseignants auraient donc davantage d'effet que l'établissement à proprement parler, quand on oppose les établissements REP et témoins.

\section{SYNTHÈSE ET DISCUSSION}

L'originalité de la présente recherche par rapport aux études antérieures portant sur l'efficacité du REP genevois est liée à sa méthodologie : nous avons tenté de nous rapprocher d'une comparaison quasi expérimentale en constituant un groupe d'établissements témoins (groupe de contrôle) aussi semblables que possible du groupe d'établissements REP (groupe expérimental). Globalement, les analyses n'ont pas 
Tableau 1. Répartition de la variance dans les trois niveaux étudiés en $2 \mathrm{e}, 4^{\mathrm{e}}$ et $6^{\mathrm{e}}$ années primaires

\begin{tabular}{|c|c|c|c|c|}
\hline & $\begin{array}{c}\text { Part de variance } \\
\text { intraclasse (niveau } \\
\text { élèves) }\end{array}$ & $\begin{array}{c}\text { Part de variance } \\
\text { interclasse (niveau } \\
\text { classes) }\end{array}$ & $\begin{array}{l}\text { Part de variance inter- } \\
\text { établissements (niveau } \\
\text { établissements) }\end{array}$ & Total \\
\hline $\begin{array}{l}\text { Compréhension de l'écrit } \\
\text { ( } 21 \text { pts) }\end{array}$ & $83,6 \%$ & $13,9 \%$ & $2,5 \%$ & $100 \%$ \\
\hline Mathématiques (24 pts) & $89,6 \%$ & $9,4 \%$ & $0,9 \%$ (n. s.) & $100 \%$ \\
\hline \multicolumn{5}{|c|}{$4^{\mathrm{e}}$ année primaire ( $\mathrm{N}=931$ élèves, 52 classes et 21 établissements) } \\
\hline $\begin{array}{l}\text { Compréhension de l'écrit } \\
\text { ( } 25 \text { pts) }\end{array}$ & $92,6 \%$ & $4,1 \%$ & $1,8 \%$ & $100 \%$ \\
\hline Français structuration (33 pts) & $96,4 \%$ & $6,6 \%$ & & $100 \%$ \\
\hline $\begin{array}{l}\text { Compréhension de l'écrit } \\
\text { (21 pts) }\end{array}$ & $97,6 \%$ & $2,0 \%$ & $0,4 \%$ (n. s.) & $100 \%$ \\
\hline Français structuration (65 pts) & $93,8 \%$ & $4,2 \%$ & $2,0 \%$ & $100 \%$ \\
\hline Mathématiques (32 pts) & $92,8 \%$ & $5,2 \%$ & $2,0 \%$ & $100 \%$ \\
\hline
\end{tabular}

Note : «n. s. » est utilisé pour « non significatif ».

permis de mettre en évidence des différences de résultats entre les deux types d'établissements (REP et témoins). En revanche, nous avons réitéré le constat classique, observant en moyenne une meilleure réussite (c'est-à-dire de meilleures performances aux évaluations externes organisées par le canton) des élèves scolarisés dans les établissements hors REP par rapport à ceux qui fréquentent les établissements REP et les établissements témoins. Toutefois, lorsque l'on contrôle la proportion d'élèves de milieux défavorisés, la situation est plus nuancée : certains établissements REP présentent une valeur ajoutée positive comparable à ce que l'on peut voir dans certains établissements hors REP. De même, on a pu observer des différences de résultats parmi les classes REP, celles-ci s'expliquant notamment par leur composition, mais probablement aussi par d'autres facteurs liés aux pratiques pédagogiques ${ }^{20}$ ou à la collaboration des enseignants, à l'implication des parents, etc. Ces deux derniers éléments n'ont pas été pris en compte dans notre étude, mais on connaît leur rôle dans le développement des connaissances et l'amélioration des acquis des élèves, notamment dans des classes de type REP (Moisan \& Simon, 1997). II s'agit d'ailleurs de deux éléments considérés dans la mise en œuvre du REP (dans le projet d'établissement pour ce qui concerne les enseignants et éducateurs, dans le cas du renforcement entre l'école et les familles pour les relations avec ces dernières). Les analyses multiniveaux réalisées confirment le poids des caractéristiques individuelles, en particulier du niveau initial, ce qui était attendu. Par ailleurs, il faut regretter que ces analyses indiquent que la part de variance expliquée par les niveaux supérieurs (classe et surtout établissement) est non significative. II semble donc qu'il faille conclure à l'absence d'effet spécifique du REP sur les acquis des élèves. À décharge de cette absence d'effet, il faut rappeler que l'évaluation des effets du REP mis en place à Genève pose un problème méthodologique particulier dû au fait que les établissements sont entrés dans le dispositif à des moments différents, et donc que les mesures propres à cette action sont installées depuis des durées variables selon les établissements ${ }^{21}$. Par ailleurs, il importe de souligner que les mesures spécifiques au REP se sont généralisées pour la plupart à l'ensemble des établissements primaires (directions, conseils et projets d'établissement notamment) en parallèle à la mise en place du REP.

Quoi qu'il en soit, il nous faut reconnaître la difficulté de mettre en évidence des effets du dispositif REP et, plus généralement, des politiques de discrimination positive. Car ces résultats confirment ceux d'autres 
études françaises (Armand \& Gille, 2006 ; Kherroubi \& Rochex, 2004 ; Rochex, 2006) et européennes (Demeuse, Frandji, Greger et al., 2008) portant sur la même thématique. D'après Rochex (2008), ce bilan mitigé serait dû notamment au fait que les pays mettent en œuvre des politiques d'éducation prioritaire (dans un souci d'améliorer l'équité) en même temps que d'autres mesures de politiques socioéconomiques pouvant avoir un effet néfaste sur la situation sociale et économique des populations visées par ces politiques d'éducation prioritaire. D'autres raisons sont également invoquées pour expliquer ce bilan : des données d'évaluation peu fiables ou insuffisantes, la difficulté d'isoler les effets des nombreuses mesures mises en place, l'évolution des PEP elles-mêmes, etc.

Par ailleurs, des variations entre classes présentant les mêmes caractéristiques sociodémographiques ont été relevées. Ce constat peut être mis en parallèle avec les résultats de Meuret (1994) attestant d'effets au niveau du climat des établissements ou des classes, ou encore au niveau de la socialisation des élèves. Une observation répétée à Genève par Jaeggi et Osiek $(2007,2008)$ suggère qu'il pourrait être utile d'insérer ce type de variables (amélioration du climat à l'intérieur de l'établissement, développement de la socialisation, etc.) dans les analyses multiniveaux. D'autres éléments, liés aux pratiques pédagogiques, nous paraissent également indispensables à prendre en compte. En effet des études françaises (voir, entre autres, Bautier, 2002 ; Chartier, 1992) ont montré la particularité des pratiques nécessaires à des élèves de milieux défavorisés ou d'origine étrangère, qui mobilisent les mêmes processus cognitifs que leurs camarades mais ont des besoins spécifiques. Chartier (1992) relève leur besoin d'apprendre l'école pour apprendre à l'école (Chartier, 1992, p. 137 ; cf. également Kherroubi \& Rochex, 2004 ; Rochex, 2008). Avant de conclure définitivement à l'absence d'effet du dispositif REP, il semble utile de s'intéresser à ces variations entre classes et de tenter de modéliser des dimensions liées spécifiquement au fonctionnement des classes et des établissements. Rappelons, dans le même esprit, que le rapport de Moisan et Simon (1997) sur les ZEP analysait de façon très fine ce qui caractérisait les ZEP « performantes ». II donnait également des pistes à suivre dans sa conclusion, notamment sur les pratiques pédagogiques efficaces: donner du sens aux activités, s'adapter aux capacités des élèves sans diminuer le degré d'exigence, ne pas restreindre l'univers des possibles (niveau de langue, centres d'intérêt), différencier les approches, développer l'activité intellectuelle, varier les situations d'apprentissage, utiliser des "détours " mais sans perdre de vue l'objectif et préciser la progression des compétences. De tels paramètres devraient pouvoir être pris en compte dans les futures évaluations du REP genevois. Car il importe de prendre en considération que cette politique y est récente. II est donc indispensable de poursuivre l'effort d'évaluation afin de voir au bout de quelques années si les mesures entreprises ont un effet quelconque. Les concepteurs du REP genevois se sont inspirés des expériences antérieures d'autres pays en mettant en place un certain nombre de mesures considérées comme favorables à l'amélioration des conditions d'enseignement : augmenter le taux d'encadrement (moins d'élèves par classe), garantir une certaine stabilité des enseignants, introduire des directions d'établissement et des projets d'établissement afin de fixer des objectifs à atteindre, renforcer les liens avec les familles (notamment grâce aux éducateurs), développer des partenariats (avec les communes en particulier), etc. Par contre, des mesures touchant de plus près les pratiques pédagogiques n'ont pas été clairement définies, puisqu'elles relèvent de l'autonomie des enseignants et des établissements.

Par ailleurs, comme nous le notions dans une autre étude (Dutrévis \& Crahay, 2009), même en l'absence d'effet des politiques de discrimination positive, il n'est guère envisageable de les supprimer sans réflexion approfondie sur les enjeux sociaux. Selon nous, malgré les résultats décevants des évaluations empiriques, il paraît important de poursuivre l'effort, ne serait-ce que pour apporter une aide nécessaire aux enseignants et aux élèves dans des écoles dont l'environnement est difficile. Ces établissements REP présentent en effet des caractéristiques particulières qui justifient des mesures spécifiques non seulement au niveau des conditions d'enseignement (moins d'élèves par classe, stabilité des enseignants dans l'établissement, projet d'école, éducateur, direction d'établissement), mais également au niveau de pratiques pédagogiques prenant en compte le rapport au savoir spécifique de ces élèves (cf. notamment les travaux de Charlot, Bautier \& Rochex, 1992 ; Bautier, Charlot \& Rochex, 2000). On peut se demander si la mesure d'effet ne suppose pas la prise en compte d'éléments plus qualitatifs liés à la dynamique interne aux écoles et aux pratiques des enseignants. II serait intéressant de faire le suivi de cette étude au bout de 5 ou 10 ans, à la fois de manière quantitative, mais également en réalisant des études de cas des établissements REP qui obtiennent de bons résultats aux évaluations externes. 
Anne Soussi

anne.soussi@etat.ge.ch

République et canton de Genève (Suisse), Service de la recherche en éducation

Christian Nidegger

République et canton de Genève (Suisse), Service de la recherche en éducation
Marion Dutrévis

Université de Genève (Suisse), Développement, apprentissage et intervention en situations scolaires

Marcel Crahay

Université de Genève (Suisse), Développement, apprentissage et intervention en situations scolaires et Université de Liège (Belgique), Approches psychopédagogiques et apprentissages scolaires

\section{NOTES}

1 Cf. la revue de littérature sur les pays nord-américains, l'Angleterre et la France (Dutrévis \& Crahay, 2009).

2 Cette étude fait le point sur ces politiques dans huit pays européens.

3 Dans cette logique d'élèves à besoins spécifiques, on peut isoler aussi bien les enfants de réfugiés, les enfants handicapés, les élèves en difficulté ou encore les élèves " doués".

4 La première étude PISA, en 2000 , a remis cette problématique au goût du jour en montrant la proportion relativement importante d'élèves présentant de faibles compétences en lecture à la fin de l'école obligatoire et le poids important de l'origine socioéconomique. À Genève, comme dans d'autres cantons romands, des mesures ont été alors mises en place pour réduire les différences entre élèves, notamment en introduisant la scolarité obligatoire à 4 ans ainsi que des dispositifs d'enseignement continué et de motivation à la lecture.

5 Ces mesures ont d'abord été introduites dans une école pilote se caractérisant par des conditions particulièrement défavorables forte proportion d'élèves provenant de milieux défavorisés (écart de $10 \%$ par rapport aux autres écoles défavorisées) et d'élèves allophones. La population du quartier dans lequel se trouvait l'école avait connu une forte évolution négative avec la présence d'un foyer de réfugiés et d'un foyer de mères en difficulté. Face aux difficultés croissantes rencontrées dans leur enseignement, les maîtres ont demandé de l'aide aux autorités pour résoudre les tensions et travailler sur certains thèmes tels que la violence et l'interculturalité. C'est ainsi qu'ont été introduites les prémices du réseau d'enseignement prioritaire. Par ailleurs, à Genève, depuis une dizaine d'années, le taux de chômage a connu une augmentation importante (passant de $4 \%$ en 2000 à près de $8 \%$ en 2005 et de $2 \%$ à $4 \%$ pour l'ensemble de la Suisse) et le nombre de personnes recevant une aide sociale a beaucoup progressé. Le taux de précarité y est de manière globale plus important en référence à l'ensemble de la Suisse et particulièrement concentré dans certains quartiers ou communes du canton.

6 L'école primaire débute officiellement à 6 ans et s'achève à 12 ans. Elle comprend deux années d'école enfantine, non obligatoires jusqu'à la rentrée 2011-2012. Une grande majorité d'enfants fréquente la $1^{\text {re }}$ année d'école enfantine dès 4 ans. Bon nombre d'entre eux ont également fréquenté auparavant des institutions de la petite enfance (crèches et jardins d'enfants principalement). Quant à l'éducation préscolaire, le contexte genevois se situe entre celui des pays anglo-saxons, avec la présence d'institutions de la petite enfance fréquentées par un nombre variable d'enfants, et celui de la France (généralisation de la maternelle avec une perspective scolarisée), la grande majorité d'élèves entrant à l'école enfantine, jusque-là non obligatoire, à 4 ans.

7 Depuis la rentrée 2011-2012, cette politique s'est étendue au secondaire inférieur dans 4 établissements sur les 20 établissements que compte le réseau.

8 Suite aux expériences d'autres pays comme la France notamment (cf. le rapport de Moisan \& Simon, 1997), il a été montré que la stabilité de l'équipe enseignante était une condition favorable à l'efficacité des dispositifs d'éducation prioritaire.

9 Cet axe ne sera pas développé dans cet article. Pour en savoir plus, on se reportera aux deux rapports précités.
10 Cette observation a été réalisée au moyen de tests provenant d'une batterie d'instruments mis au point par Saada (2006) et expérimentée sur une population tout-venant du même âge, tests auxquels ont été ajoutés deux sous-tests du WPPSI-III (Wechsler preschool and primary scale of intelligence, instrument standardisé évaluant différents aspects de l'intelligence), celui du vocabulaire et celui des « matrices" (pris ici au sens de « raisonnement »). Les connaissances ou compétences observées dans le domaine du langage portaient sur les éléments suivants: compréhension de consignes orales, lecture et identification de mots, segmentation phonologique, identification et écriture de lettres, lecture et écriture du prénom et vocabulaire ; dans le domaine des mathématiques, sur les connaissances de la suite des nombres, le comptage et le dénombrement, la lecture et l'écriture des nombres, la constitution de la suite des nombres, la comparaison de collections, les " matrices".

11 Parmi les règles de vie de la classe figuraient des items tels qu'« écouter les autres", "prendre en compte les règles de jeu [de société] " ou " demander l'autorisation pour sortir ou prendre la parole ". La mesure des relations avec les enseignants et de l'autonomie portait sur des éléments tels qu'« écouter l'adulte ", "se débrouiller seul » ou " choisir des activités "; celle des relations avec les pairs portaient notamment sur la collaboration. Enfin l'attitude des élèves par rapport au travail scolaire était observée grâce à de nombreux items, parmi lesquels " comprendre les consignes ", " faire ce qui est demandé » ou « aller jusqu'au bout de l'activité ».

12 II est alors probable que l'effet de la fréquentation d'une institution de la petite enfance soit inclus dans le score initial mesuré en début d'année scolaire.

13 Cette dernière catégorie est donnée à titre indicatif étant donné son statut particulier. Les élèves peuvent être considérés comme en retard scolaire principalement pour deux raisons : avoir redoublé ou avoir commencé l'école avec du retard, étant donné leur maîtrise insuffisante du français. Par ailleurs, si le redoublement est basé sur des normes communes à tous les établissements, la politique interne aux établissements peut varier.

14 Précisons qu'il est un peu artificiel de constituer un tel groupe puisque les établissements remplissant ce type de conditions se trouvent justement dans le REP. De plus, parmi ces 7 établissements, trois sont entrés dans le REP depuis.

15 Ces degrés correspondent respectivement au CE1, au CM1 et à la 6e française. Précisons que depuis la rentrée 2011-2012, une nouvelle numérotation des années scolaires est entrée en vigueur avec l'harmonisation de la scolarité en Suisse (HarmoS) : les années sont numérotées en continu de la $1^{\text {re }}(4$ ans) à la 11e (15 ans, fin de la scolarité obligatoire). Les évaluations externes sont données à l'ensemble des élèves de ces degrés en français communication (compréhension de l'écrit et production écrite), français structuration (vocabulaire, orthographe, grammaire et conjugaison), mathématiques et allemand (dès la $4 \mathrm{e}$ année primaire) à la fin de l'année. Ces épreuves ont une visée certificative puisqu'elles participent à la certification des élèves dans le cycle ou le degré suivant.

16 On peut se demander si le temps écoulé (trois ans pour la première école, deux ans voire une année pour les autres) n'est 
pas trop court pour observer des effets. De ce fait, il conviendra de rester prudent dans l'interprétation des résultats et de réitérer régulièrement ce type d'évaluation pour une analyse à moyen terme des effets du REP.

17 Pour obtenir la valeur ajoutée, nous avons calculé à nouveau pour chaque établissement ce que devrait être la réussite attendue de l'établissement, compte tenu de la proportion des trois catégories socio-économiques (milieu défavorisé, moyen et favorisé) et de la réussite des élèves de chacune de ces catégories.

18 Cette variable n'est pas pertinente en $2^{\mathrm{e}}$ année primaire, étant donné qu'il n'y a pratiquement pas d'élèves ayant redoublé ou en avance scolaire.
19 II n'y a pas de score initial en $2^{\mathrm{e}}$ année primaire puisque c'est la première année pour laquelle les élèves sont soumis à une évaluation cantonale.

20 On a pu observer dans un échantillon de classes des différences de scores aux diverses composantes de l'évaluation (par exemple, pour les mathématiques, domaine du nombre ou de la géométrie ou, pour le français structuration, vocabulaire, grammaire, orthographe ou conjugaison) pouvant être attribuées à des centrations plus ou moins marquées des enseignants sur les domaines pris en compte.

21 À noter que cette étude s'inscrit dans un programme de recherche dans lequel d'autres analyses seront menées, dont l'une permettant de voir l'évolution des effets année après année pour chaque établissement puisque, comme évoqué plus haut, le REP s'est constitué en différentes phases.

\section{BIBLIOGRAPHIE}

ARMAND A. \& GILLE B. (2006). La contribution de l'éducation prioritaire à l'égalité des chances des élèves. Rapport IGEN et IGAENR n 2006-076 au ministre de l'Éducation nationale, de l'Enseignement supérieur et de la recherche. Paris : Ministère de l'Éducation nationale, de l'Enseignement supérieur et de la recherche.

BARA F., GENTAZ É. \& COLÉ P. (2008). « Littératie précoce et apprentissage de la lecture : comparaison entre des enfants à risque, scolarisés en France dans des réseaux d'éducation prioritaire, et des enfants de classes régulières ". Revue des sciences de l'éducation, vol. 34, no 1, p. 27-45.

BAUTIER É. (2002). " L'enseignement en ZEP et les recherches en didactique du français ". Revue française de pédagogie, n 140, p. 53-64.

BAUTIER É., CHARLOT B. \& ROCHEX J.-Y. (2000). "Entre apprentissages et métier d'élève : le rapport au savoir ". In A. van Zanten (dir.), L'école, l'état des savoirs. Paris: La Découverte, p. 179-188.

BRESSOUX P. (1994). "Estimer et expliquer les effets des classes: le cas des acquisitions en lecture ". Mesure et évaluation en éducation, vol. 17, n० 1, p. 75-94.

BRESSOUX P. (2009). "Des contextes scolaires inégaux : effets-établissements, effets-classes et effets du groupe de pairs ". In M. Duru-Bellat \& A. van Zanten (dir.), Sociologie du système éducatif. Les inégalités sociales. Paris : PUF, p. 131-148.

CHARLOT B., BAUTIER É. \& ROCHEX J.-Y. (1992). École et savoir dans les banlieues... et ailleurs. Paris : Armand Colin.

CHARTIER A.-M. (1992). "Questions d'apprentissage ». Cahiers pédagogiques, n॰309, p. 19-21.

CHAUVEAU G. (2000). Comment réussir en ZEP. Vers des zones d'excellence pédagogique. Paris : Retz.

CRAHAY M. (2000). L'école peut-elle être juste et efficace? Bruxelles: De Boeck.

CRAHAY M. (2006). Un bilan des recherches processusproduit. Genève : Université de Genève, coll. " Carnets des sciences de l'éducation ".

CRAHAY M. \& DUTRÉVIS M. (2012). Prévention de l'échec scolaire par l'éducation préprimaire. Genève : Université de Genève, coll. "Carnets des sciences de l'éducation".
DEMEUSE M., FRANDJI D., GREGER D. \& ROCHEX J.-Y. (2008). Les politiques d'éducation prioritaire en Europe. Conceptions, mises en œuvre, débats. Lyon : INRP.

DUMAY X. \& DUPRIEZ V. (2009). L'efficacité dans l'enseignement. Bruxelles: De Boeck.

DURU-BELLAT M., LE BASTARD-LANDRIER S., PIQUÉE C. \& SUCHAUT B. (2004). "Tonalité sociale du contexte et expérience scolaire des élèves au lycée et à l'école primaire ». Revue française de sociologie, vol. $45, n^{\circ} 3$, p. 441-468.

DUTRÉVIS M. \& CRAHAY M. (2009). " Les politiques de discrimination positive : un bilan impossible ". In G. Chapelle \& M. Crahay (dir.), Réussir à apprendre. Paris : PUF, p. 59-73.

HUTMACHER W. (1993). Quand la réalité résiste à la lutte contre l'échec scolaire. Cahier no 36 du service de la recherche sociologique. Genève : Département de l'instruction publique, de la culture et du sport. En ligne: http://www.geneve.ch/recherche-education/ publications/cahiers.asp (consulté le 25 mars 2012).

JAEGGI J.-M. \& OSIEK F. (2007). Mise en place d'un dispositif-pilote dans le groupe scolaire Gros-Chêne/ Tattes. Premier rapport intermédiaire. Année scolaire 2006-07. Rapport de recherche. Genève : Département de l'instruction publique, de la culture et du sport, service de la recherche en éducation.

JAEGGI J.-M. \& OSIEK F. (2008). Extension du réseau d'enseignement prioritaire à six nouvelles écoles genevoises. Deuxième rapport intermédiaire. Année scolaire 2007-2008. Rapport de recherche. Genève : Département de l'instruction publique, de la culture et du sport, service de la recherche en éducation.

KHERROUBI M. \& ROCHEX J.-Y. (2004). « La recherche en éducation et les ZEP en France. Deuxième partie : apprentissages et exercice professionnel en ZEP : résultats, analyse, interprétations ". Revue française de pédagogie, no 146, p. 115-190.

MEURET D. (1994). «L'efficacité de la politique des zones d'éducation prioritaire dans les collèges ". Revue française de pédagogie, no 109, p. 41-64.

MEURET D. (2000). "Les politiques de discrimination positive en France et à l'étranger ". In A. van Zanten (dir.), L'école, l'état des savoirs. Paris : La Découverte, p. 112-120. 
MOISAN C. \& SIMON J. (1997). Les déterminants de la réussite scolaire en zone d'éducation prioritaire. Rapport IGEN et IGAEN au ministre de l'Éducation nationale. Paris : La Documentation française.

PERRENOUD P. (1984). La fabrication de l'excellence scolaire. Genève : Droz.

PIQUÉE C. (2010). «Pratiques enseignantes envers les élèves en difficulté dans des classes à efficacité contrastée ". Revue française de pédagogie, n 170 , p. 43-60.

ROCHEX J.-Y. (2006). «Les "zones d'éducation prioritaire" (ZEP) ? Quel bilan ? " Les temps modernes, no 637$638-639$, p. 219-253

ROCHEX J.-Y. (2008). « Les politiques d'éducation prioritaire en Europe, d'un "âge" et d'un pays à l'autre ". In M. Demeuse, D. Frandji, D. Greger \& J.-Y. Rochex (dir.), Les politiques d'éducation prioritaire en Europe. Conceptions, mises en œuvre, débats. Lyon : INRP, p. 409-451.

ROCHEX J.-Y. (2010). "Les "trois âges" des politiques d'éducation prioritaire : une convergence européenne ? » In C. Ben Ayed (dir.), L'école démocratique. Vers un renoncement politique ? Paris: Armand Colin, p. 94-108.

SAADA E. (2006). Connaissances initiales des enfants à l'entrée à l'école. Rapport de recherche. Genève : Département de l'instruction publique, de la culture et du sport, service de la recherche en éducation.

SIROTA R. (1993). "Le métier d'élève ». Revue française de pédagogie, no 104, p. 85-108.

SOUSSI A. \& NIDEGGER C. (2010). Le réseau d'enseignement prioritaire à Genève : quels effets sur les acquis des élèves quelques années après ? Rapport de recherche. Genève : Département de l'instruction publique, de la culture et du sport, service de la recherche en éducation.

SOUSSI A., GUIGNARD N., HAYOZ E. \& NIDEGGER C. (2008). Mise en place du REP : effets sur les compétences des élèves. Rapport de recherche. Genève : Département de l'instruction publique, de la culture et du sport, service de la recherche en éducation. 